\title{
Research
}

Catrin P Penn-Jones, Chris Papadopoulos, Gurch Randhawa and Zeeshan Asghar

\section{Improving access to organ donor registration in general practice:}

a feasibility study

\begin{abstract}
Background

Organ donor registration helps guide decision making for families. UK general practice provides the facility to register on the NHS Organ Donor Register, but only to new patients. An intervention was developed to present a registration opportunity to existing patients in this setting.
\end{abstract}

Aim

To assess the feasibility and acceptability of an organ donation intervention implemented in UK general practice.

\section{Design and setting}

The intervention ran in a large practice in Luton in the UK, for 3 months in 2018. A single practice feasibility study was conducted using an embedded experimental mixed methods design

\section{Method}

Staff were trained to ask patients in consultations if they wished to join the register and leaflets and posters were displayed in the waiting room. Data on feasibility and acceptability were captured using SystmONE questionnaires, surveys, and focus groups.

\section{Results}

Over 3 months, in $12.4 \%$ of face-to-face consultations, patients were asked if they would like to join the register (812 of 6569), and 244 (30.0\%) of these patients joined the register. Common reasons staff did not ask patients were due to telephone consultations, lack of time, and it not being appropriate. Nurses and healthcare assistants performed prompted choice more than doctors $(23.4 \%, 17.1 \%$, and $1.6 \%$ respectively). Certain clinic types, such as phlebotomy or routine clinics, facilitated asking compared to those where patients presented with unknown or more serious issues.

\section{Conclusion}

The intervention was found to be feasible and acceptable by some staff and patients. Feasibility criteria were met; therefore, the intervention can progress to further testing.

\section{Keywords}

decision making; feasibility studies; organ donation, primary care.

\section{INTRODUCTION}

In the UK, there is a shortage of organs for donation. Between March 2018 and April 2019, 1600 people donated their organs, resulting in 3951 transplants. ${ }^{1}$ However, during the same period, 6077 people were on the transplant waiting list. ${ }^{1}$ This shortage of organs requires addressing to help increase the number of life-saving transplants carried out in the UK. In order to do this, it is important to examine the facilitators of positive donation decisions. A key part of the organ donation pathway is the role of families in the process, who ultimately decide whether organ donation should proceed. The academic literature has also repeatedly found that expressing a wish to donate through registration is vital to families giving consent to organ donation. ${ }^{2-7}$ This is demonstrated by the fact that $46 \%$ of donors had registered their wish to donate on the NHS Organ Donor Register (ODR) in 2018 to 2019. ' With only $38 \%$ of the population registered on the NHS ODR, however, interventions are required to increase the number of people expressing their intention to donate in this way.

In 2018, a consultation was held on the transition of England from an opt-in system of organ donation consent to an opt-out system. ${ }^{8}$ Based on the responses received, England will transition to an opt-out system of consent from Spring 2020. ${ }^{8}$ Wales have implemented an opt-out system of consent

CP Penn-Jones, PhD, researcher:

C Papadopoulos, $\mathrm{PhD}$, principal lecturer in public health; G Randhawa, PhD, professor of diversity in public health; director, Institute for Health Research, University of Bedfordshire, Luton. Z Asghar, partnership development manager, NHS Blood and Transplant, London.

\section{Address for correspondence}

Catrin P Penn-Jones,MRC Epidemiology Unit,

University of Cambridge School of Clinical Medicine, Box 285 Institute of Metabolic Science, Cambridge since 2015, introducing an opt-out register andmarketing campaigntotargetawareness of the change in legislation. ${ }^{9}$ Rates of organ donation consent have increased since its introduction. ${ }^{10}$ However, the increase in these rates cannot be attributed to opt-out policy alone, instead to the opt-out policy combined with interventions and structural changes implemented alongside it. ${ }^{10,11}$ Critically, families will still be consulted on whether donation should proceed, and if they do not consent, donation will not take place. This means that registration on the NHS ODR is still a vital part of the consent process. Therefore, it is important to examine other opt-in interventions that can be implemented alongside optout, maximising public organ donation awareness.

General practice currently offers an opportunity for new patients to register on the NHS ODR via a tick box on their new patient registration form. ${ }^{12}$ Staff at practices then enter the details of those wishing to register directly onto the NHS ODR via computer systems. This, however, is only offered to new patients. There is an opportunity to target existing patients in general practice, and based on this, a new intervention was designed to fill this gap.

In order to develop this intervention, a systematic review investigating organ donation interventions in primary care or general practice settings was used as a basis. Ten studies were found, and only four of these

Biomedical Campus, Cambridge CB2 OQQ, UK

Email: catrin.penn-jonesamrc-epid.cam.ac.uk

Submitted: 4 November 2019; Editor's response: 25 November 2019; final acceptance: 8 December 2019

\section{CBritish Journal of General Practice}

This is the full-length article (published online 5 May 2020) of an abridged version published in print. Cite this version as: Br J Gen Pract 2020; DOI: https://doi.org/10.3399/bjgp20X709601 


\section{How this fits in}

Nowhere currently in the UK, are people able to sign up to the NHS Organ Donor Register verbally. The opportunity to do this was tested in a single UK GP practice, where staff asked their patients in consultations if they would like to join. This intervention was successfully conducted, demonstrating that it is feasible and acceptable for some staff and some patients. The intervention also demonstrated promise for increasing registration rates.

were peer-reviewed journal articles. Despite a limited evidence base, consistently positive findings were found for active interventions, as opposed to passive conducted in this setting..$^{13}$ Active interventions were those in which patients were presented with an opportunity to register or discuss organ donation. Passive interventions, on the other hand, were when materials were placed in practices and patients would have to approach, view, or pick these up on their own initiative. These findings provided a basis for an active intervention to be developed.

The technique used in this study's intervention was an active method, called prompted choice, where patients were asked in consultations if they wished to join the NHS ODR. At present, nowhere in the UK provides the opportunity for people to register face to face. In the US, however, this opportunity is provided to visitors to the Driving Motor Vehicles Offices. This technique has proved successful in recruitment to state registries by many authors, and prompted choice aims to replicate this success. ${ }^{14-16}$ Importantly, two studies from the systematic review, conducted in the UK, found significant barriers to implementation of interventions in general practice, particularly: available time, resources, and competing priorities..$^{13}$ This study, therefore, aimed to assess the feasibility and acceptability of an organ donation intervention implemented in UK general practice, with the results expected to inform refinements to the intervention and data collection materials prior to effectiveness testing.

\section{METHOD}

Intervention

The main intervention component to be tested for feasibility and acceptability, was prompted choice. Development of the intervention used a detailed method called intervention mapping (IM), ${ }^{17}$ and was underpinned by the IIFF model of organ donation registration. ${ }^{18}$ The IIFF model recommends four components be included in interventions: an Immediate sign-up opportunity; Information provision, particularly concerning common myths; Focused engagement with the intervention; and Favourable activation, where the patient should be in a positive affective state. To supplement prompted choice, and fulfil the IIFF model, two additional components were included as part of the intervention package': staff training and providing leaflets and posters in the waiting room.

\section{Participants and setting}

One large practice in Luton, UK, participated in this study. The practice is located in an area in the top $10 \%$ of the most deprived areas nationally, with $45.3 \%$ of Luton residents classified as 'non-white'; ${ }^{19}$ compared with $13.8 \%$ in England. ${ }^{20}$ All staff were invited to attend the training; clinical staff conducted prompted choice, and staff voluntarily participated in post-intervention data collection. All patients who visited the practice during the intervention period could be exposed to the intervention. Training was conducted in March 2018, prompted choice ran from 5 April to 9 July 2019, and postintervention data was collected from 9 July to 20 September 2018.

\section{Materials and procedure}

Training. Training was designed by NHS Blood and Transplant, and adapted by a specialist nurse in organ donation, NHS Blood and Transplant representative, and a researcher. It was delivered in two sessions in March 2018. Training development and evaluation will be discussed in a separate paper.

Prompt and questionnaire. To facilitate staff conducting prompted choice, a prompt and questionnaire were developed on the practice computer system (SystmONE). The prompt appeared only to trained clinical staff at the beginning of all consultations had with patients aged $>18$ years. Staff were then able to select 'No - patient will not be asked', and record reasons for this (lack of time, not appropriate, clinician personal beliefs, and other), or 'Yes - patient will be asked'. The prompted choice questionnaire contained an eligibility question (that is, does the patient have the capacity to consent), then staff could enter the patient response to prompted choice, which were 


\section{Box 1. Prompted choice questionnaire for trained clinical staff at the beginning of the consultation}

\begin{tabular}{|c|c|c|c|c|}
\hline \multirow[t]{7}{*}{ Yes } & Any organs or tissues & & & \\
\hline & Selected organs & Heart & & \\
\hline & & Liver & & \\
\hline & & Kidneys & & \\
\hline & & Corneas & & \\
\hline & & Lungs & & \\
\hline & & Pancreas & & \\
\hline \multicolumn{5}{|l|}{$\begin{array}{l}\text { Unsure - Patient } \\
\text { will think about it }\end{array}$} \\
\hline \multicolumn{5}{|l|}{$\begin{array}{l}\text { Do not ask patient } \\
\text { again }\end{array}$} \\
\hline \multirow{10}{*}{$\begin{array}{l}\text { Patient believes } \\
\text { they are already } \\
\text { on the register }\end{array}$} & Would the patient & Yes & Any organs or tissues & \\
\hline & like to re-register? & & Selected organs & Heart \\
\hline & & & & Liver \\
\hline & & & & Kidneys \\
\hline & & & & Corneas \\
\hline & & & & Lungs \\
\hline & & & & Pancreas \\
\hline & & No - Patient & & \\
\hline & & would not like & & \\
\hline & & to re-register & & \\
\hline \multirow[t]{4}{*}{$\begin{array}{l}\text { Patient was not } \\
\text { asked }\end{array}$} & $\begin{array}{l}\text { Not appropriate for } \\
\text { consultation }\end{array}$ & & & \\
\hline & Lack of time & & & \\
\hline & $\begin{array}{l}\text { Clinician personal } \\
\text { beliefs }\end{array}$ & & & \\
\hline & $\begin{array}{l}\text { Other reason, please } \\
\text { specify }\end{array}$ & & & \\
\hline
\end{tabular}

based on the new patient registration form (see Box 1; further information available from authors on request). Following prompted choice, the prompt was switched off for future appointments if the patient responded that they would like to join the NHS ODR, or they believed they were already on the register. If staff selected the option 'do not ask patient again', this would also switch off the prompt. The number of staff consultations conducted in the 3-month period was also collected, counted manually by a researcher during weekly monitoring visits, and separated into face-to-face consultations and telephone consultations.

\section{Leaflets and posters}

NHS Blood and Transplant provided leaflets and posters for display in the practice during the 3-month period. The intervention was monitored weekly by the researcher who checked for positioning of these and restocked them when necessary. Leaflets were counted at the end of the intervention period to establish how many were taken over 3 months.

\section{Post intervention data collection}

Focus groups and online surveys were conducted to examine staff views of the intervention. Four focus groups took place in the practice between July and August 2018 during staff lunch breaks. These were separated according to staff group (GPs, nurses and healthcare assistants, receptionists and administrative staff). One focus group was conducted with five nurses and healthcare assistants, and one focus group with four administrative and reception staff. Focus groups were attempted for GPs, however, on two separate occasions, only one participant attended, resulting in two GPs being interviewed. Participants read a participant information sheet and signed a consent form prior to participation. Topic guides were created and adhered to by the facilitator and staff debriefed post focus group session (further information available from authors on request). Separate topic guides were used for clinical staff who participated in prompted choice and those who were administrative and reception staff. Following focus groups, all participating staff were sent a link to a qualitative online survey (further information available from authors on request), hosted on Qualtrics, via email by the practice manager. This contained an information sheet and consent page, 13 open questions based on the focus group topic guide, and a debrief page.

Patient views were captured using an online survey. Those, for whom a prompted choice questionnaire was completed, were recruited to a mixed methods online survey via text message sent by the practice. Not all patients that were sent a text message had prompted choice conducted, due to the ability of staff to select 'patient not asked' in the prompted choice questionnaire. The survey was also hosted on Qualtrics, and patients were presented with an information sheet, consent page, three demographic questions, three closed questions and nine open questions, followed by a debrief page (further information available from authors on request). Of the 743 patients who received a text message, 36 completed the online survey. Respondents had a mean age of $43,22 \%$ were male, $(n=8) 86 \%(n=31)$ were white, and $22 \%(n=8)$ responded that prompted choice was not conducted in their consultation.

\section{Analysis}

Quantitative analyses were conducted on SPSS version 23, and Qualitative analysis 
on NVivo version 11. Quantitative analysis was descriptive, examining prompt and questionnaire data from SystmONE, as well as the number of leaflets taken. Qualitative framework analysis was conducted on focus group data, and staff online survey responses. A descriptive thematic framework approach was taken, with an a priori framework specified and subsequent a posteriori themes added to the framework. The a priori framework consisted of contextual levels of primary care intervention implementation, as described by Lau et al lexternal context, organisational context, professional context and intervention context) ${ }^{21}$ as well as the IIFF model of organ donation. ${ }^{18} \mathrm{~A}$ list of preliminary themes was created, based on first impressions. These were integrated into the framework, the data were then coded to the framework, and charts populated with summaries recorded for each framework cell. The patient online survey was analysed using only descriptive thematic analysis. This is due to the brief responses provided by patients to the online survey questions.

\section{RESULTS}

\section{Prompted choice questionnaire}

Of the 12 recruited, 10 clinical staff completed prompted choice on more than one occasion in their consultations. Two GPs did not conduct prompted choice on any occasion, nor did they complete any form of data collection. A total of 10144 consultations were conducted during the 3-month period, 6569 face-to-face consultations, and 3575 telephone consultations. Prompted choice was conducted by staff in none of the telephone consultations, and in 812 $(12.4 \%)$ of all face-to-face consultations

\section{Table 1. A breakdown of patient responses to prompted choice and reasons why prompted choice was not conducted}

\section{Patient preference}

Yes - any organs or tissues

Yes - selected organs or tissues

Patient believes they are already on NHS ODR

Patient is unsure

Do not ask patient again

Total

\section{Patient not asked ( $N=2772$ )}

Reason patient not asked

Lack of time

Not appropriate for consultation

Lack of time and other

Other

NHS ODR = NHS Organ Donor Register . conducted by participating staff members. Doctors conducted prompted choice on 46 out of 2875 face-to-face consultations (1.6\%), nurses on 472 occasions out of 2014 face-to-face consultations (23.4\%), and healthcare assistants on 296 occasions out of 1734 face-to-face consultations (17.1\%).

Of the 812 patients asked, 244 joined the NHS ODR (30.0\%). Table 1 shows the full breakdown of patient responses to prompted choice. Based on the responses in Table 1, 352 patients $143.3 \%$ of those asked) were added to the NHS ODR for the first or second time lby wishing to re-register) as a result of the intervention.

Prompted choice reached 187 black, asian and minority ethnic (BAME) patients, and 326 non-BAME patients. Of these patients, 39 BAME patients joined the NHS ODR for the first time, $20.9 \%$ of those asked. Ethnicity was not specified for 299 patients who received prompted choice. The average age of patients targeted was 50.2 years (with a standard deviation of 17.6, a minimum of 18 , a maximum of 91 , a median of 50 , and a mode of 67 years).

\section{Prompted choice feasibility questionnaire}

The feasibility questionnaire was completed 2906 times, and, on 134 occasions, only partially completed. During face-to-face consultations, a feasibility questionnaire was completed 1690 times, and, on 1051 occasions, prompted choice was stated not to be conducted due to a telephone consultation. Lack of time was stated as the reason for not conducting prompted choice on $34.0 \% \quad(n=943)$ of feasibility questionnaires and not appropriate for consultation on $18.8 \% \quad(n=520)$ questionnaires. On 43 occasions, a language barrier was specified, and other reasons were suggested on less than 10 occasions each (for example, data entry error, home visit, lack of capacity, or over eligible age limit).

\section{Leaflets and posters}

One hundred leaflets were taken during the 3-month intervention period by patients. Leaflets and posters were displayed as per protocol throughout the intervention period and were only restocked on one occasion.

\section{Staff focus groups and online survey}

External contextual factors were less prominent in the focus groups discussions, and only once was the opt-out policy mentioned by staff. Predominantly, staff discussed GP settings generally, media exposure to organ donation, registration 
through the Driving Vehicle Licensing Agency, and that organ donation is not a normal thing to discuss in society:

In the general world of general practice, we've got the knowledge and the expertise, and, especially with a little bit of training, I think that's fine.' (Focus group [FG], GP)

Well, normally it's a no-no, you don't talk about things like that, do you?' (FG, administrative and reception!

At the organisation contextual level, staff discussed that they could not conduct prompted choice during telephone consultations, that it was easier to conduct prompted choice with weekend patients lpatients who attend the GP practice at the weekend, that is, those who are more likely to work Monday to Fridayl, that there is a culture of collaboration and prioritising patient care in the practice, and that some consultations were more suitable for conducting prompted choice than in others. This latter theme was strong throughout, as those clinics that are 'routine', for example. phlebotomy or practice nurse sessions, were more suitable for prompted choice. Clinics where the patient could present with a serious issue, particularly those conducted by GPs, as well as those where nurses did not know why patients were visiting, were more challenging:

'You've got to listen to the patient, you've got to examine the patient, you've got to figure out what's wrong, you've then got to, you know, prescribe, do the [prescription], then give the advice, so that's completely different to a practice nurse session ... or maybe even your phlebotomy session.' (FG, nurse and healthcare assistants)

Professional contextual factors included a wide variety of perspectives around prompted choice. Views among staff were polarised, with some lthose who conducted it more often), believing it to be very acceptable and easy to conduct, and others (those conducting it less often), believing it to be challenging to conduct.

Overall, however, staff had positive attitudes to prompted choice, believing it to be a good idea, and to be congruent with their professional role in most cases.

At the intervention contextual level, lack of time during consultations was the barrier discussed most often, alongside whether it was appropriate to ask all patients, and concerns over negatively impacting the patient relationship:
I would be happy to continue asking my patients if intervention was reintroduced as I feel it is a tremendously worthwhile [and] beneficial process to contribute towards. (Staff online survey)

I still think general practice would probably be the best place for these things to happen and it's a real shame if we cannot do this. (FG, GP)

'It depends on the patients really ... because if they were really ill, I didn't, I wouldn't bring it up because I think that's not appropriate.' (FG, GP)

'The time was quite limited [because], you know, like you said, they've come in and they want to start telling you what's wrong. (FG, nurse and healthcare assistants)

Staff also reported problems with the SystmONE prompt and questionnaire, mainly that it was too long and complex. Staff had mixed views on the usefulness of leaflets and posters, with some using them to start the prompted choice process, and others believing patients did not read them.

Finally, regarding the IIFF model, providing information as part of prompted choice was discussed by all staff verbally and also through the leaflets, and most staff were comfortable with this. Mixed views were discussed concerning an immediate registration opportunity, with some staff believing it was a good idea, and others that it was better for patients to go away and think about it. Fewer discussions were had on the topic of focused engagement, however, staff mentioned that some patients wanted to start talking about their problem immediately, and then wanted to leave once they were finished, indicating that patients may not have been focused on the topic of organ donation during prompted choice.

Favourable activation, however, was discussed at length, with some staff concerned about patients being distressed by the topic, and it not being appropriate. However, some discussed that they did not have any instances where a patient was distressed.

\section{Patient online survey}

Of the 36 patients who completed the online survey, 21 patients were asked by a nurse or healthcare assistant, seven were asked by a doctor or GP, and eight were not asked at all. 12 patients noticed the leaflets and posters displayed in the practice, and 22 patients did not. 
Responses to the open questions showed most patients believed the intervention to be acceptable and appropriate in a general practice setting:

\% went in to have my smear test and was asked at the end. I thought it was a great idea to ask me whilst in the room for a medical reason.' (Patient Online Survey)

As long as asked once and not pressured, I'm not bothered. I am already a donor. (Patient Online Survey)

'This is probably the most appropriate place to be approached. '(Patient Online Survey)

'This is the best place for the conversation in my view.' (Patient Online Survey)

Some patients described that it might be annoying, and one patient described feeling pressured to sign up. Patients, on the whole, did not find the leaflets and posters useful. This was further illustrated by recommendations provided by the patients, including providing leaflets in the waiting rooms when these were already provided.

Ok. Its same as being bombarded with tv ads. Annoying.' (Patient Online Survey)

'[Leaflets are] useful as information reminders, but little influence on decision. The major influence for me, on deciding this sort of thing, is speaking with someone. (Patient Online Survey)

\section{DISCUSSION}

\section{Summary}

This study investigated the feasibility and acceptability of an organ donation intervention in general practice. Staff in the participating practice aimed to ask patients during consultations if they would like to join the NHS ODR. Staff were trained to do this, and leaflets and posters were displayed in the practice. Over 3 months, 812 patients experienced prompted choice, and 43.3\% joined the NHS ODR, with 244 joining the NHS ODR for the first time (30.0\%), and 108 joining for the second time (13.3\%). Overall, the intervention was found to be feasible to conduct with some patients by some staff members. It was also acceptable to staff and patients as long as patients, for whom it is not appropriate, were not asked. On $34.0 \%$ of occasions where a patient was not asked this was reported to be due to a lack of time, and $18.8 \%$ due to it not being appropriate. Mixed results were found for the usefulness of leaflets and posters, as only 100 were taken when 6569 face-toface consultations occurred in the practice during the prompted choice period.

\section{Strengths and limitations}

A strength of this research was that an in-depth examination of feasibility and acceptability was conducted using a number of mixed methods approaches. This allowed for the collection of a breadth and depth of data, including quantitative reasons for why the intervention was not conducted, and in depth exploration of these reasons during focus groups. Additionally, the intervention shows it has promise for providing a new opportunity for people to join the NHS ODR, as patients were asked in $12.4 \%$ of consultations during 3 months, and $30.0 \%$ of these joined. The intervention was also developed using a rigorous and detailed method, intervention mapping, ${ }^{17}$ and was underpinned by the IIFF model of organ donation registration, ${ }^{18}$ which could be a reason for its success. A number of stakeholders participated in the design and delivery of the intervention, including the participating practice, NHS Blood and Transplant, the patient participation group of the practice, and a specialist nurse in organ donation. Including stakeholders in this process has been found to positively impact the success of interventions in previous literature..$^{22}$

There are limitations to this research, however. Recruitment to staff focus groups, online survey, and the patient online survey was lower than anticipated. Additionally, the participation of only one practice, although results are promising, does not allow for transferability between practices. Therefore, further investigations of feasibility and acceptability in multiple practices are required before firm conclusions can be made. Further challenges were found, due to the constraints of the practice computer system to develop the prompt and questionnaire. Finally, a detailed examination of intervention cost was omitted from this study, due to resource and expertise limitations in the study team.

\section{Comparison with existing literature}

The findings support previous literature that active organ donation interventions in primary care are superior to passive. ${ }^{13}$ Only 100 leaflets were taken during the 3 months la passive technique), with no guarantee patients would register on the NHS ODR. Whereas 244 patients signed up for the first time over 3 months using prompted choice, an active intervention. However, these findings are not conclusive. This is 


\section{Funding}

This study forms part of a PhD at the University of Bedfordshire. The PhD is match funded by both the University of Bedfordshire and NHS Blood and Transplant.

\section{Ethical approval}

Ethical approval was granted by Health Research Authority on 11 December 2017 (IRAS ID: 230702), favourable opinion by the NHS Research Ethics Committee on 3 November 2017 (17/LO/1361) and favourable opinion by the Confidentiality Advisory Group on 8 December 2017 (17/ CAG/0169). University of Bedfordshire Institute for Health Research Ethics Approval was granted on 20 November 2017 (IHREC800). Several non-substantial amendments were approved by the Health Research Authority (23 February 2018, 1 March 2018, 2 May 2018 and 2July 2018). Trial registration: International Standard Randomised Controlled Trial Number ISRTN44530504. Registration on 26 September 2017.

\section{Competing interests}

The authors declare that they have no competing interests

\section{Acknowledgments}

The authors would like to offer their thanks to both the University of Bedfordshire and NHS Blood and Transplant for funding this study. The authors would also like to thank the GP Practice who agreed to take part in this study; particularly the practice manager, lead GP and patient participant group. Finally, the authors would like to express their gratitude to the Specialist Nurse in Organ Donation who conducted the training as part of this study.

\section{Discuss this article}

Contribute and read comments about this article: bjgp.org/letters

also only the second intervention targeting organ donation to be designed using intervention mapping to the best of the authors' knowledge..$^{23}$ This research adds to the knowledge base using this technique in this context, and supports that interventions designed using it can be successfully implemented. Finally, compared to the extensive barriers to interventions and challenges to implementing them expressed for primary care settings, ${ }^{24-29}$ this research shows a relatively successful rate of conducting brief verbal interventions in general practice.

\section{Implications for research and practice}

The implications of this research are that it is possible for staff, in some circumstances, to ask patients, in consultations, if they wish to join the NHS ODR. Staff and patients find this intervention feasible and acceptable during some consultations, which means it can be tested further. The finding that nurses and healthcare assistants were able to perform prompted choice more often than GPs is particularly pertinent for further testing. Combined with the qualitative findings, reasons for this are likely to be that GPs see patients with more serious and complex issues than do nurses and healthcare assistants.
Whether prompted choice was 'appropriate' for all patients was discussed as a key theme, and this could explain why GPs conducted it less than other staff members, because it is less appropriate for patients visiting a GP with a more serious issue. Additionally, nurses and healthcare assistants discussed that prompted choice was easier to conduct in more 'routine' clinics, such as phlebotomy, where they knew in advance why the patient was visiting. This has important implications for testing the intervention further. It is possible that the intervention could be restricted to routine consultations such as screening, phlebotomy, or health checks. However, it is recommended that further feasibility testing is conducted to explore this finding, and examine whether it occurs in multiple practices, or is specific to the single practice that participated in the present study. A feasibility randomised controlled trial in multiple practices could fulfil this requirement, to examine transferability of the intervention between them. Additionally, it could obtain statistics to inform a sample size calculation and establish intervention costs. Finally, this could also gather more data on patient and staff acceptability, particularly of GPs, as only two participated in the focus groups. 


\section{REFERENCES}

1. NHS Blood and Transplant. Organ Donation and Transplantation. Activity Report 2018/19. 2019. https://nhsbtdbe.blob.core.windows.net/umbraco-assetscorp/16537/organ-donation-and-transplantation-activity-report-2018-2019.pdf laccessed 05 Mar 2020

2. Hulme W, Allen J, Manara AR, et al. Factors influencing the family consent rate for organ donation in the UK. Anaesthesia 2016; 71(9): 1053-1063.

3. Sque M, Walker W, Long-Sutehall T, et al. Bereaved donor families' experiences of organ and tissue donation, and perceived influences on their decision making. J Crit Care 2018; 45: 82-89.

4. Walker W, Broderick A, Sque M. Factors influencing bereaved families' decisions about organ donation: an integrative literature review. West J Nurs Res 2013; 35(10): 1339-1359.

5. Chandler JA, Connors M, Holland G, Shemie SD. "Effective" Requesting: A scoping review of the literature on asking families to consent to organ and tissue donation. Transplantation 2017; 101(5S Suppl 1): S1-S16.

6. Ralph A, Chapman JR, Gillis J, et al. Family perspectives on deceased organ donation: thematic synthesis of qualitative studies. Am J Transplant 2014; 14(4): 923-935.

7. de Groot J, Vernooii-Dassen M, Hoedemaekers C, et al. Decision making by relatives about brain death organ donation: an integrative review. Transplantation 2012; 93(12): 1196-2111.

8. NHS Blood and Transplant. Organ donation laws. How the law affects you https://www.organdonation.nhs.uk/faq/is-organ-donation-law-changing/ (accessed 27 Apr 2020)

9. Shaw D. Presumed evidence in deemed consent to organ donation. J Intensive Care Soc 2018;19(1): 2-3.

10. Noyes J, McLaughlin L, Morgan K, et al. Short-term impact of introducing a soft opt-out organ donation system in Wales: before and after study. BMJ Open 2019; 9(4): e025159.

11. Carr-Hill RA. Organ donation in Wales [Letter]. Br J Gen Pract 2018; DOI: https://doi.org/10.3399/bjgp18X694961

12. NHS. Form GMS1. Family doctor services registration. 2019. https://uww.gov. uk/government/publications/gms1 laccessed 05 Mar 2020)

13. Jones CP, Papadopoulos C, Randhawa G. Primary care interventions to encourage organ donation registration: a systematic review. Transplant Rev 2017: 31(4): 268-275.

14. Rodrigue JR, Krouse J, Carroll C, et al. A Department of Motor Vehicles intervention yields moderate increases in donor designation rates. Prog Transplant 2012; 22(1): 18-24

15. Zaramo CE, Morton T, Yoo JW, et al. Culturally Competent Methods to Promote Organ Donation Rates Among African-Americans Using Venues of the Bureau of Motor Vehicles. Transplant Proc 2008; 40(4): 1001-1004.

16. Harrison TR, Morgan SE, Di Corcia MJ. Effects of information, education, and communication training about organ donation for gatekeepers: clerks at the
Department of Motor Vehicles and organ donor registries. Prog Transplant 2008; 18(4): 301-309.

17. Eldredge LKB, Markham CM, Ruiter RAC, et al. Planning health promotion programs: an intervention mapping approach. 4th ed. San Francisco, CA: John Wiley \& Sons; 2016

18. Siegel JT, Alvaro EM. A Dawning Recognition of Factors for Increasing Donor Registration: The IIFF Model. In: Understanding Organ Donation: Applied Behavioral Science Perspectives. Malden, MA: Wiley-Blackwell; 2010; 313-330.

19. Office for National Statistics. Nomis. nomis. Official labour market statistics. QS201EW. Ethnic group. Ethnic Group by Rural Urban. https://www.nomisweb. co.uk/census/2011/QS201EW/view/1946157201?rows=c ethnicid\&cols=rural urban (accessed 10 Mar 2020).

20. Gov.uk. Population of England and Wales. Ethnicity facts and figures. UK population by ethnicity. 1. Main facts and figures 2018. https://www.ethnicityfacts-figures.service.gov.uk/uk-population-by-ethnicity/national-and-regionalpopulations/population-of-england-and-wales/latest (accessed 6 Mar 2020).

21. Lau R, Stevenson F, Ong BN, et al. Achieving change in primary care - causes of the evidence to practice gap: systematic reviews of reviews. Implement Sci 2016;11: 40

22. O'Cathain A, Croot L, Sworn K, et al. Taxonomy of approaches to developing interventions to improve health: a systematic methods overview. Pilot Feasibility Stud 2019; 5(1): 41.

23. Squires JE, Grimshaw JM, Taljaard M, et al. Design, implementation, and evaluation of a knowledge translation intervention to increase organ donation after cardiocirculatory death in Canada: a study protocol. Implement Sci 2014; 9(1): 80.

24. Mason V, Shaw A, Wiles N, et al. GPs' experiences of primary care mental health research: A qualitative study of the barriers to recruitment. Fam Pract 2007; 24(5): 518-25

25. Yallop JJ, Mcavoy BR, Croucher JL, et al. Primary health care researchessential but disadvantaged. Med J Aust 2006; 185(2): 118-120.

26. Michalec B, Fagan HB, Rahmer B. Primary care practices' perceived constraints to engaging in research: the importance of context and 'Flow'. Prim Health Care Res Dev 2014; 15(1): 58-71.

27. Brodaty $\mathrm{H}$, Gibson LH, Waine ML, et al. Research in general practice: a survey of incentives and disincentives for research participation. Ment Health Fam Med 2013; 10(3): 163-173

28. Bower $\mathrm{P}$, Wallace $\mathrm{P}$, Ward $\mathrm{E}$, et al. Improving recruitment to health research in primary care. Fam Pract 2009; 26(5): 391-397.

29. Hummers-Pradier E, Scheidt-Nave C, Martin H, et al. Simply no time? Barriers to GPs' participation in primary health care research. Fam Pract 2008; 25(2): $105-112$ 